\title{
Apoyo social percibido y factores sociodemográficos en relación con los síntomas de ansiedad, depresión y estrés en universitarios chilenos
}

\author{
Ana Barrera-Herrera, Mariajosé Neira-Cofré, Paola Raipán-Gómez, \\ Patricio Riquelme-Lobos y Bertha Escobar \\ Universidad Católica de Temuco, Temuco, Chile
}

\begin{abstract}
Perceived social support and socio-demographic factors in relation to symptoms of anxiety, depression and stress in Chilean university students. Research with university students shows a high prevalence of mental disorders, so it is necessary to study the impact of protective variables such as social support. The objective of this study was to determine the influence of perceived social support and the socio-demographic factors gender and socio-economical level on symptomatology related to depression, anxiety, and stress. In the framework of a non-experimental and cross-sectional design, 449 undergraduate students answered the Depression, Anxiety and Stress Scales (DASS-21) and the Multidimensional Scale of Perceived Social Support (MSPSS). Results showed that perceived social support was negatively associated with psychopathological symptoms, and that family support is the strongest predictor of mental health symptomatology. These findings indicate that family social support is important to Chilean university students and plays a protective role against the symptoms of anxiety, depression and stress in this stage in life.
\end{abstract}

Keywords: emerging adulthood; perceived social support; depression; anxiety; stress.

Resumen: Las investigaciones con universitarios evidencian alta prevalencia de trastornos mentales por lo que es necesario estudiar el impacto de variables protectoras como el apoyo social. El objetivo del estudio fue determinar la influencia del apoyo social percibido y de los factores sociodemográficos de género y nivel socioeconómico en la sintomatología depresiva, ansiosa y de estrés. En el marco de un diseño no experimental y transversal, 449 estudiantes universitarios respondieron las Escalas de Depresión, Ansiedad y Estrés (DASS-21) y la Escala Multidimensional de Apoyo Social Percibido (MSPSS). Los resultados mostraron que el apoyo social percibido se asocia de forma inversa a los síntomas psicopatológicos y que el apoyo familiar es el mejor predictor ante la aparición de sintomatología de salud mental. Los resultados confirman que los jóvenes chilenos otorgan importancia al apoyo social familiar, el cual cumple un rol protector ante los síntomas de ansiedad, depresión y estrés en esta etapa vital.

Palabras clave: Adultez emergente; apoyo social percibido; depresión; ansiedad; estrés.

\section{Introducción}

Los jóvenes entre los 18 y 29 años atraviesan una etapa evolutiva conocida como adultez emergente (Arnett, 2000), cuya característica más distintiva es que quienes

Recibido: 31 de enero 2019; aceptado: 09 de mayo 2019

Correspondencia: Dra. Ana Barrera-Herrera, Departamento de Psicología, Universidad Católica de Temuco, Manuel Montt 056, Temuco, Chile. Correo-e: abarrera@uct.cl

Agradecimientos: Investigación patrocinada por el Proyecto PAI 77170108 CONICYT. transitan por ella no se ven como adolescentes, pero tampoco sienten que han alcanzado la adultez, aplazando hitos como el matrimonio, la paternidad, la independencia financiera o la salida del hogar hasta una edad cercana a los 30 años (Arnett, 2000, 2008). Si bien la descripción y teorización de la adultez emergente surgió en Estados Unidos, el interés por esta etapa de vida se ha expandido rápidamente por Latinoamérica (Dutra-Thomé, 2013; Facio y Micocci, 2003; Fierro y Moreno, 2007; Pérez, Cumsille, y Martínez, 2008). En Chile, un estudio tuvo por propósito indagar la presencia de la adultez emergente en universitarios, un grupo que tran- 
sita por el rango de edad descrito por esta etapa de vida (Barrera-Herrera y Vinet, 2017). Esta investigación constató la existencia de la adultez emergente en este grupo de jóvenes, puesto que las características centrales de la teoría son reconocidas y vivenciadas por los universitarios chilenos (exploración de la identidad, inestabilidad, gran optimismo y posibilidades, estar centrado en sí mismos, y sentirse "en el medio" entre la adolescencia y la adultez). Además, el estudio reporta evidencia de aspectos de carácter cultural propios de la adultez emergente como, por ejemplo, la gran importancia que los vínculos familiares y sociales tienen para estos universitarios (Barrera-Herrera y Vinet, 2017).

En los últimos años, las investigaciones en salud mental han propiciado centrar la atención en los universitarios, pues los estudios tanto a nivel nacional como internacional señalan que las características de la etapa, junto con los desafíos que enfrentan los jóvenes en la universidad tales como el manejo de la autonomía, asumir responsabilidades académicas, responder a expectativas personales y familiares, pueden desencadenar la aparición de trastornos o sintomatología de salud mental (Arnett, Žukauskienè, y Sugimura, 2014; Baader et al., 2014; Micin y Bagladi, 2011).

En cuanto a la sintomatología depresiva, ansiosa y de estrés, las investigaciones realizadas en Chile con universitarios se han centrado en estudiar la prevalencia de los trastornos más comunes tales como depresión, ansiedad, estrés, trastornos de la conducta alimentaria, o consumo de sustancias y riesgo suicida, encontrándose los dos primeros como los más prevalentes en estos jóvenes (Antúnez y Vinet, 2013; Baader et al., 2014; Micin y Bagladi, 2011).

Al realizar un análisis detallado de la evidencia disponible en estos tres cuadros sintomatológicos, es posible observar que la prevalencia reportada en universitarios es preocupante. Estudios internacionales señalan que la prevalencia de sintomatología depresiva supera el 20\% (Pego-Pérez, Río-Nieto, Fernández, y Gutiérrez-García, 2018) y que los universitarios pertenecientes a estratos socioeconómicos bajos presentan mayores niveles de depresión (Ferrel, Celis, y Hernández, 2011). En Chile, las cifras en sintomatología depresiva son similares a las mencionadas por el estudio de Pego-Pérez et al. (2018), variando éstas según la intensidad de los síntomas (Micin y Bagladi, 2011) y el género de los sujetos, presentando las mujeres mayor prevalencia en depresión (Antúnez y Vinet, 2013; Baader et al., 2014).

En lo que respecta a sintomatología ansiosa, las cifras a nivel nacional e internacional siguen una tendencia similar a las referidas a la depresión. Por ejemplo, un estudio con universitarios estadounidenses indicó prevalencias cercanas al 11.5\% (Chandavarkar, Azzam, y Mathews,
2007). Asimismo, un estudio con universitarios colombianos encontró que quienes pertenecen a estratos socioeconómicos bajos presentaban mayor sintomatología ansiosa (Arrieta, Díaz, y González, 2014). En el contexto chileno, estudios con universitarios informan de cifras cercanas al 20\% (Antúnez y Vinet, 2013; Micin y Bagladi, 2011), encontrando que las mujeres presentaban mayor sintomatología ansiosa que los hombres (Antúnez y Vinet, 2013).

Por último, respecto al estrés, los estudios informan de una variada y alta prevalencia. Investigaciones con estudiantes mexicanos reportaron que la percepción de estrés fue del $60.7 \%$, existiendo una mayor percepción de éste en mujeres (Halgravez, Salinas, Martínez, y Rodríguez, 2016). Además, se señala que aquellos universitarios que se perciben con una condición socioeconómica baja informan de un nivel de estrés más alto y frecuente que aquellos que se perciben con un nivel socioeconómico medio y alto (Belhumeur, Barrientos, y Retana-Salazar, 2016). Por su parte, en Chile, los estudios con universitarios han referido prevalencias de estrés sobre el $30 \%$, siendo esta sintomatología significativamente mayor en las mujeres (Barraza et al., 2015; Marty, Lavín, Figueroa, Larraín, y Cruz, 2005).

Como puede observarse, existen altas cifras de prevalencia de sintomatología depresiva, ansiosa y de estrés en universitarios y las variables sociodemográficas de género y nivel socioeconómico constituyen factores de riesgo para la presencia de este tipo de sintomatologías. Sin embargo, frente a estas altas prevalencias también es importante distinguir variables protectoras de la salud mental como el apoyo social percibido. En concreto, el apoyo social constituye un factor determinante en la salud mental, ya que se ha comprobado que posee efectos protectores sobre la salud y el bienestar de los individuos y cumple la función de amortiguar la patología o malestar psicológico (Aranda y Pando, 2013; Organización Mundial de la Salud [OMS], 2003).

Si bien existen diversas definiciones de apoyo social, para los fines de la presente investigación se entiende el apoyo social percibido como la valoración subjetiva de la calidad del apoyo social existente de diversas fuentes externas, las cuales pueden clasificarse en tres: el apoyo social percibido de la familia, de los amigos, y de otras personas significativas (Zimet, Dahlem, Zimet, y Farley, 1988).

Las investigaciones disponibles respecto a la relación entre el apoyo social percibido y la salud mental señalan que los universitarios europeos al dejar el hogar, y por ello alejarse de sus familias nucleares para realizar sus estudios superiores, no cuentan con el apoyo social de tipo familiar, lo que puede influir en el desarrollo de síntomas depresivos (Stroebe, Van Vliet, y Hewstone, 2002). En el contexto chileno, una investigación realizada con univer- 
sitarios de la capital del país demostró que los estudiantes que mantienen un apoyo social satisfactorio, tanto de los amigos como de la familia presentan baja sintomatología depresiva (Dávila, Ruiz, Moncada, y Gallardo, 2011).

Respecto a la relación entre el apoyo social y la ansiedad, un estudio realizado con universitarios españoles mostró que vivir sin la familia y, por lo tanto, no contar con ese tipo de apoyo social durante el periodo universitario puede ser un factor asociado al riesgo de presentar sintomatología ansiosa (Balanza, Morales, y Guerrero, 2009). Por su parte, en Chile, una investigación destacó el aporte amortiguador efectuado por el apoyo social, el cual actúa en momentos en los que se produce un aumento de la sintomatología ansiosa en los universitarios, como, por ejemplo, en periodo de exámenes (Dávila et al., 2011).

Finalmente, en relación con el apoyo social y el estrés, una investigación con universitarios colombianos arrojó resultados que demuestran la asociación entre la disfunción familiar y otros factores académicos y sociales en la presencia de sintomatología de estrés, informando que aquellos estudiantes que percibían un pobre apoyo de sus amigos mostraban niveles más altos de sintomatología de estrés (Arrieta, Díaz, y González, 2013). Asimismo, un estudio con universitarios venezolanos demostró que, a mayor estrés académico, menor apoyo social percibido (Feldman et al., 2008). Por su parte, en Chile no hemos encontrado estudios que hayan estudiado la relación entre el apoyo social y el estrés en la etapa universitaria.

Como se observa, a pesar de que existen algunos estudios sobre apoyo social y salud mental en universitarios (Balanza et al., 2009; Dávila et al., 2011), estos presentan algunas limitaciones. Por ejemplo, no consideran la dimensión subjetiva del apoyo social, no detallan la asociación específica entre estas variables y no aportan evidencia sobre el posible papel predictor del apoyo social percibido en sus distintas facetas (familia, amigos y otros significativos) en relación con la presencia de sintomatología de salud mental en población universitaria.

Por lo tanto, considerando los antecedentes y las limitaciones de los estudios previos, cabe preguntarse ¿cómo influye el apoyo social percibido en la presencia de sintomatología depresiva, ansiosa y de estrés en universitarios chilenos y cómo se comporta esta sintomatología según el género y el nivel socioeconómico? Se desprenden tres hipótesis sustentadas en los antecedentes anteriormente expuestos: (1) Las universitarias presentarían puntuaciones más altas en sintomatología depresiva, ansiosa y/o de estrés respecto a sus pares de género masculino, (2) Los universitarios que pertenecen a niveles socioeconómicos más bajos mostrarían puntajes más altos en sintomatología depresiva, ansiosa y/o de estrés respecto a los universitarios que pertenecen a niveles socioeconómicos más altos, y (3) A mayor apoyo social percibido, se presentaría menor sintomatología depresiva, ansiosa y/o de estrés.

El objetivo general del estudio consistió en examinar la influencia del apoyo social percibido y de factores sociodemográficos (género y nivel socioeconómico) en la presencia de sintomatología depresiva, ansiosa y de estrés en universitarios chilenos. Los objetivos específicos fueron: (1) Comparar la sintomatología depresiva, ansiosa y de estrés en grupos diferenciados por género y nivel socioeconómico, (2) Analizar si existen diferencias en el apoyo social percibido (amigos, familia y otros significativos) según factores sociodemográficos (género y nivel socioeconómico) y (3) Determinar el grado de asociación $\mathrm{y} / \mathrm{o}$ predicción del apoyo social percibido (amigos, familia y otros significativos) respecto a la presencia de sintomatología depresiva, ansiosa y de estrés.

\section{Método}

\section{Participantes}

La muestra estuvo constituida por 449 estudiantes chilenos de la Universidad de Tarapacá y Universidad Católica de Temuco, las cuales se ubican en las ciudades de Arica y Temuco $(55.2 \%$ y $44.8 \%$ de estudiantes, respectivamente), seleccionados mediante un muestreo no probabilístico por conveniencia. Los criterios de inclusión para participar en el estudio fueron: encontrarse entre el rango de edad de 18 a 29 años y tener nacionalidad chilena.

El $66.8 \%$ de los participantes eran mujeres y el promedio de edad del grupo total fue 20.82 años $(D T=$ 2.33) con un rango de edad de la muestra comprendido entre 18 y 29 años. La mayoría de los participantes procedía de zonas urbanas $(87.9 \%)$, mientras que un porcentaje menor lo hacía de zonas rurales (11.4\%). El $61.7 \%$ de los jóvenes vivía con uno o ambos padres mientras que el 38.3\% vivía de manera independiente. En su mayoría, eran solteros (99.1\%), y solo un $23.2 \%$ de la muestra estudiaba y trabajaba.

En relación con el nivel socioeconómico, esta variable se calculó bajo el método ESOMAR, que es la forma adaptada y validada para medir los niveles socioeconómicos en encuestas e investigaciones en Chile (ADIMARK, 2000). Esta medida se basa en dos variables: el nivel de educación alcanzado por el jefe/jefa de hogar y la categoría ocupacional de esta persona, arrojando seis niveles, que van desde un nivel socioeconómico bajo a alto. Para fines de esta investigación, se recategorizó esta variable en tres niveles obteniendo así que un $30.1 \%$ de 
la muestra se ubicaba en el nivel socioeconómico bajo, un $50.8 \%$ en el nivel medio, y el $11.8 \%$ en un nivel socioeconómico alto. Finalmente, ante a la pregunta “¿Sientes que has alcanzado la adultez?”, un $65.9 \%$ estaba de acuerdo con que en "algunos aspectos sí, mientras que en otros no". Las características sociodemográficas de la muestra se observan en más detalle en la Tabla 1.

Tabla 1. Características sociodemográficas de la muestra

\begin{tabular}{|c|c|c|}
\hline Variables sociodemográficas & $n$ & $\%$ \\
\hline \multicolumn{3}{|l|}{ Sexo } \\
\hline Masculino & 146 & 32.5 \\
\hline Femenino & 300 & 66.8 \\
\hline Perdidos & 3 & .7 \\
\hline \multicolumn{3}{|l|}{ Procedencia } \\
\hline Urbana & 395 & 87.9 \\
\hline Rural & 51 & 11.4 \\
\hline Perdidos & 3 & .7 \\
\hline \multicolumn{3}{|l|}{ Vives con } \\
\hline Uno o ambos padres & 277 & 61.7 \\
\hline Independiente de sus padres & 172 & 38.3 \\
\hline \multicolumn{3}{|l|}{ Estado civil } \\
\hline Soltero/a & 445 & 99.1 \\
\hline Casado/a & 3 & .7 \\
\hline Divorciado/a & 1 & .2 \\
\hline \multicolumn{3}{|l|}{ Estudio y trabajo } \\
\hline Sí & 104 & 23.2 \\
\hline No & 344 & 76.6 \\
\hline Perdidos & 1 & .2 \\
\hline \multicolumn{3}{|l|}{ Nivel Socioeconómico } \\
\hline Bajo & 135 & 30.1 \\
\hline Medio & 228 & 50.8 \\
\hline Alto & 53 & 11.8 \\
\hline Perdidos & 33 & 7.3 \\
\hline \multicolumn{3}{|l|}{ Sentirse adulto } \\
\hline Sí & 57 & 12.7 \\
\hline No & 90 & 20.1 \\
\hline En algunos aspectos sí, en otros no & 296 & 65.9 \\
\hline Perdidos & 6 & 1.3 \\
\hline
\end{tabular}

\section{Diseño y procedimiento}

Esta investigación es de carácter cuantitativo, con un diseño no experimental de tipo transversal, un alcance descriptivo-correlacional, con el objetivo principal de determinar la influencia del apoyo social y de factores sociodemográficos en la presencia de sintomatología depresiva, ansiosa y de estrés en universitarios chilenos. Los participantes fueron contactados a través de informantes claves de las universidades de interés (profesores o directores de carrera). Todos los participantes firmaron un consentimiento informado, en el cual se dio a conocer el objetivo del estudio, se explicitó la voluntariedad de la participación y el carácter anónimo y confidencial del uso de la información. Este documento fue visado y aprobado por el Comité de Ética de la Investigación de la Universidad Católica de Temuco, al igual que todos los procedimientos descritos en esta investigación. Una vez contactada la muestra, el equipo de investigación procedió a la aplicación de los instrumentos durante el horario de clases, previa autorización del docente a cargo de la asignatura.

\section{Instrumentos}

Cuestionario Sociodemográfico. Cuestionario de auto-reporte construido por el equipo de investigación, el cual contiene preguntas para recoger datos que permitieron caracterizar la muestra como edad, género y nivel socioeconómico.

Escalas de Depresión, Ansiedad y Estrés [Depression, Anxiety and Stress Scales] (DASS-21; Lovibond y Lovibond, 1995). Se utilizó la versión chilena validada y estandarizada con muestra universitaria (Antúnez y Vinet, 2012). Este instrumento de auto-informe tiene como propósito medir la sintomatología depresiva, ansiosa y de estrés, así como el grado y la frecuencia en que se han experimentado estos estados durante la última semana. Consta de 21 ítems con cuatro alternativas de respuesta en formato Likert, las cuales van desde 0 ("No describe nada de lo que me pasó o sentí en la semana") hasta 3 ("Sí, esto me pasó mucho, o casi siempre"). Cada escala contiene siete ítems y la puntuación por subescala puede variar entre 0 y 21 puntos, por lo que, a mayor puntaje obtenido, se estima que existe mayor presencia de sintomatología depresiva, ansiosa y de estrés. Para la escala de depresión el punto de corte es mayor que 5, para la escala de ansiedad mayor que $4 \mathrm{y}$, por último, para la escala de estrés, el punto de corte es mayor que 5 (Román, Santibáñez, y Vinet, 2016). En relación con la prevalencia de sintomatología obtenida en este estudio mediante el instrumento DASS-21, el $43.2 \%$ de la muestra total presentó valores superiores al punto de corte para sintomatología depresiva, un $46.9 \%$ presentó sintomatología ansiosa y un 53\% sintomatología de estrés, mientras que un $28 \%$ de los participantes presentó conjuntamente sintomatología depresiva, ansiosa y de estrés. Con respecto 
a la fiabilidad, se calculó el estadístico alfa de Cronbach: para la escala total se obtuvo un alfa de Cronbach de .92, mientras que, para sus dimensiones de depresión, ansiedad y estrés, ésta fue de $.88, .79$, y .85 respectivamente.

Escala Multidimensional de Apoyo Social Percibido [Multidimensional Scale of Perceived Social Support] (MSPSS; Zimet et al., 1988). Se utilizó la versión chilena adaptada para adultos del estudio de Ortiz y Baeza (2011). Este instrumento está conformado por 12 ítems que evalúan la percepción de apoyo social por los individuos en tres dimensiones: amigos (ítems 1, 2, 3 y 4), familia (ítems 5, 6, 7 y 8) y otros significativos (ítems 9 , 10,11 y 12); cuenta con cuatro alternativas de respuesta en formato Likert que van desde 1 ("Nunca o casi nunca") hasta 4 ("Siempre o casi siempre"). En esta investigación, el instrumento MSPSS presentó una adecuada consistencia global, obteniendo para la escala total un alfa de Cronbach de .88, mientras que la fiabilidad para las subescalas fue la siguiente: amigos $(\alpha=.87)$, familia $(\alpha=.89)$ y otros significativos $(\alpha=.90)$.

\section{Análisis estadístico}

Para responder al objetivo específico 1, esto es, comparar la sintomatología depresiva, ansiosa y de estrés en grupos diferenciados por género y nivel socioeconómico, se utilizó la prueba $t$ de Student para grupos independientes, cuyo propósito fue la comparación de los tres tipos de sintomatología según género. Luego, se utilizó la prueba de ANOVA de un factor para comparar la sintomatología depresiva, ansiosa y/o de estrés, según nivel socioeconómico. Estas mismas pruebas fueron utilizadas para responder al objetivo específico 2, a fin de examinar si existían diferencias entre el tipo de apoyo social percibido, y sus respectivas dimensiones, según género y nivel socioeconómico.

Para responder al objetivo específico 3, relacionado con determinar el grado de asociación y/o predicción del apoyo social percibido (amigos, familia y otros significativos) respecto a la presencia de sintomatología de salud mental, se calculó la $r$ de Pearson, la cual fue empleada para analizar la relación entre los tres tipos de sintomatología y los tipos de apoyo social percibido. Posteriormente se utilizó la prueba de regresión lineal múltiple por pasos sucesivos para analizar el efecto predictivo de los tipos de apoyo social percibido sobre la presencia de sintomatología depresiva, ansiosa y/o de estrés, respectivamente.

\section{Resultados}

Respecto al primer objetivo relacionado con la asociación entre la sintomatología depresiva, ansiosa y de estrés y los factores sociodemográficos (género y nivel socioeconómico recategorizado), constatamos lo siguiente: en relación con el género, la prueba $t$ de Student indicó diferencias estadísticamente significativas entre hombres y mujeres respecto a la sintomatología ansiosa $(t(444)=-5.36, p<.001)$ y de estrés $(t(444)=-5.07, p<$ $.001)$; en ambas variables las mujeres obtuvieron medias más altas que los hombres. No encontramos diferencias significativas entre hombres y mujeres respecto a sintomatología depresiva $(t(444)=-.81, n s)$. Para comparar la sintomatología de ansiedad, depresión y estrés según nivel socioeconómico recategorizado en tres niveles de la variable independiente (bajo, medio y alto), se utilizó un diseño de ANOVA de un factor, utilizando en cada análisis como variable dependiente cada una de las escalas de sintomatología. A través de estos análisis encontramos diferencias significativas para la escala de sintomatología depresiva $(F(2,415)=3.75, p<.05)$. La prueba posthoc de Bonferroni reflejó diferencias significativas entre los niveles socioeconómicos alto y medio $(p<.05)$, encontrándose las medias más altas de esta sintomatología en el nivel socioeconómico alto. Respecto a la sintomatología ansiosa, también encontramos diferencias significativas entre los tres niveles socioeconómicos analiza$\operatorname{dos}(F(2,415)=4.41, p<.05)$; la prueba post-hoc de Bonferroni indicó diferencias significativas entre los niveles socioeconómicos alto y medio $(p<.05)$, observándose nuevamente las medias más altas en el nivel socioeconómico alto. Por último, para sintomatología de estrés, no se encontraron diferencias estadísticamente significativas entre los tres niveles socioeconómicos $(F$ $(2,415)=2.01, n s)$.

En relación con el segundo objetivo, examinamos las posibles diferencias en el apoyo social percibido (amigos, familia y otros significativos) según factores sociodemográficos (género y nivel socioeconómico -bajo, medio y alto). Basándonos en la prueba $t$, no encontramos diferencias entre hombres y mujeres en los tipos de apoyo social percibido. Aplicando análisis de varianza de un factor (tres niveles socioeconómicos), encontramos un efecto significativo del nivel socioeconómico sobre el apoyo social percibido de los amigos, obteniéndose diferencias significativas entre los niveles socioeconómicos bajo y medio (prueba de Bonferroni, $p<.05$ ) (mayor apoyo social en el nivel socioeconómico medio). No encontramos diferencias relacionadas con el apoyo social de la familia ni de otras fuentes.

Finalmente, mediante el objetivo específico tres buscábamos determinar el grado de asociación y/o predicción de la variable de apoyo social percibido (familia, amigos y otros significativos) respecto a la presencia de sintomatología de salud mental (depresión, ansiedad y 
estrés). Aplicamos la prueba $r$ de Pearson, cuyos resultados se presentan en la Tabla 2. Este análisis indicó que existía una correlación estadísticamente significativa, inversa y baja, entre sintomatología depresiva y las tres dimensiones del apoyo social, a saber: apoyo social percibido de la familia, de los amigos, y de otros significativos. Asimismo, encontramos correlaciones significativas (igualmente inversas y bajas) entre la sintomatología ansiosa y apoyo social percibido en las diferentes facetas. El apoyo social correlacionó en menor grado con los síntomas de estrés (véase la Tabla 2).

Tabla 2. Correlaciones de Pearson entre sintomatología y apoyo social percibido $(N=449)$

\begin{tabular}{lccc}
\hline Variables & ASP familia & ASP amigos & ASP otros \\
\hline Depresión & $-.348^{* *}$ & $-.162^{* *}$ & $-.326^{* *}$ \\
Ansiedad & $-.287^{* *}$ & $-.104^{*}$ & $-.178^{* *}$ \\
Estrés & $-.269^{* *}$ & -.092 & $-.165^{* *}$ \\
\hline
\end{tabular}

Nota. ASP $=$ Apoyo social percibido. $p$ (bilateral $*) . p<.05 .$, $* * p<.01$;

Considerando las correlaciones indicadas arriba, se calcularon tres análisis de regresión lineal múltiple para examinar la capacidad predictiva del apoyo social (amigos, familia y otros significativos) sobre las variables dependientes de ansiedad, depresión y estrés. Se utilizó el método de pasos sucesivos, incluyendo las tres variables predictoras por cada una de las variables criterio. Los resultados de estos análisis se indican en la Tabla 3. Como puede apreciarse en la tabla, la dimensión de apoyo social familiar predijo de forma significativa las tres variables de síntomas psicopatológicos (depresión, ansiedad, y estrés), el apoyo social referido a los amigos no resultó ser una variable predictiva para ninguno de los tres tipos de síntomas, y el apoyo social referido a otros predice, junto a la familia, una porción de la varianza de la sintomatología depresiva.

\section{Discusión}

Como indicamos anteriormente, una de las variables psicosociales que ha sido vinculada como factor protector de la salud mental corresponde al apoyo social (OMS, 2003). Sin embargo, su impacto en la salud mental, específicamente en los tres problemas emocionales de mayor prevalencia en universitarios (ansiedad, depresión y estrés), ha sido poco explorado en investigaciones empíricas en Chile. En el presente estudio se ha constatado una asociación significativa entre el apoyo social y la sintomatología depresiva, ansiosa y de estrés en universitarios chilenos.

El primer objetivo se refería a la comparación de la sintomatología depresiva, ansiosa y de estrés según grupos diferenciados en función del género y el nivel socioeconómico. Constatamos que no existían diferencias significativas entre hombres y mujeres universitarios respecto a los niveles de sintomatología depresiva. Este resultado es llamativo, puesto que la mayor parte de los estudios señalan que son las mujeres las que presentan mayor sintomatología depresiva, tanto en población general como universitaria (Antúnez y Vinet, 2013; Baader et al., 2014). Algunos estudios atribuyen esta mayor vulnerabilidad en salud mental a condiciones generadas por el menor estatus social y poder de la mujer (Matud, Guerrero, y Matías, 2006). Probablemente, tales condiciones en el contexto universitario no estarían tan marcadas, puesto que la universidad es un espacio diverso donde se potencian las competencias de ambos géneros por igual (Ordorika, 2015). En contraste, sí encontramos diferencias entre hombres y mujeres en sintomatología de ansiedad y estrés, siendo las mujeres las que manifestaron mayores puntuaciones en ambas variables. Estos resultados son consistentes con los publicados por Riveros y Vinaccia-Alpi (2017), los cuales encontraron diferencias significativas en ansiedad entre hombres y mujeres (mayores puntuaciones en mujeres) en población colombiana, pero no diferencias en depresión. Así mismo, los re-

Tabla 3. Predicción de la sintomatología (depresión, ansiedad y estrés) a partir de las dimensiones de apoyo social (familia, amigos, y otros). Análisis de regresión múltiple de pasos sucesivos

\begin{tabular}{lcccc}
\hline Independiente & Dependiente & $R^{2}$ (ajustado) & $\beta$ & $t$ \\
\hline 1. ASP Familia & Síntomas depresivos & .121 & -.35 & $-7.838^{* * *}$ \\
2. ASP Familia & & .156 & -.25 & $-5.12^{* * *}$ \\
2. ASP Otros & & & -.21 & $-4.31^{* * *}$ \\
1. ASP Familia & Síntomas de ansiedad & .082 & -.28 & $-6.33^{* * *}$ \\
1. ASP Familia & Síntomas de estrés & .073 & -.27 & $-5.91^{* * *}$ \\
\hline
\end{tabular}

Nota. ASP $=$ Apoyo social percibido. $* * * p<.001$. 
sultados obtenidos sobre diferencias de género en ansiedad y estrés están en línea con la evidencia de la literatura que sugiere mayores niveles en general de estas variables en la mujer (Antúnez y Vinet, 2013; Halgravez et al., 2016; Marty et al., 2005; Sandín y Chorot, 2017).

En cuanto a las diferencias en sintomatología de salud mental según el nivel socioeconómico, encontramos diferencias significativas entre el nivel alto y el nivel medio, siendo el nivel alto el que presentaba mayores niveles. Este resultado es contradictorio con la evidencia de la literatura, ya que la mayor sintomatología se ha asociado generalmente a los niveles socioeconómicos más bajos (Arrieta et al., 2014; Ferrel et al., 2011). Este resultado inesperado podría explicarse por dos razones. En primer lugar, tal como indican Ortiz-Hernández, López-Moreno y Borges (2007), la forma de medir el nivel socioeconómico, ya sea por escolaridad, por ingresos o condiciones de vivienda, puede propiciar encontrar diferentes resultados cuando se analizan posibles diferencias en salud mental según este criterio. Como se mencionó anteriormente, en este estudio se utilizaron los criterios de nivel socioeconómico otorgados por la encuesta nacional ADIMARK. Aunque este método está validado y es ampliamente utilizado en estudios chilenos, esta forma de medición en el contexto universitario podría ser una variable imprecisa, dado que las universidades chilenas en los últimos años están utilizando el indicador de "deciles", el cual considera, para su cálculo, el ingreso per cápita familiar. Esta última medida podría ser más precisa para evaluar esta variable en este grupo debiendo explorarse qué resultados podrían obtenerse con este nuevo indicador (Galleguillos, Hernández, Sepúlveda, y Valdés, 2016).

La segunda explicación requiere de un análisis más profundo. Aun cuando innumerables estudios señalan que las vulnerabilidades en el desarrollo y salud mental han estado asociadas a los sectores socioeconómicos más pobres, se ha sugerido que existe una cifra oculta en lo que concierne a vulneraciones de derechos de niños y jóvenes del estrato socioeconómico alto debido al difícil acceso a la evaluación e investigación en contextos de salud privados, o a la dificultad para denunciar y tramitar estos casos en el sistema judicial chileno (Opazo, 2019). Por ejemplo, un estudio desarrollado en Chile revela que los niños de los estratos socioeconómicos más alto y más bajo presentaban la misma prevalencia de problemas mentales (Lecannelier, 2018), situación que podría también extrapolarse a otros grupos etarios como los adultos emergentes. Además, otros autores señalan que los niños y jóvenes de niveles socioeconómicos altos, aun cuando puedan tener recursos económicos suficientes, igualmente podrían estar viviendo una orfandad emocional ante la ausencia de fuentes afectivas, escasa interacción y falta de límites por parte de sus figuras paternas (Sinay, 2011). Sin duda este aspecto merece una investigación más extensa, con datos empíricos que permitan probar estas hipótesis en universitarios chilenos.

Continuando con el objetivo específico dos, relacionado con las posibles diferencias en el apoyo social percibido según factores sociodemográficos (género y nivel socioeconómico), se encontró que no existían diferencias significativas entre hombres y mujeres respecto al apoyo social percibido de amigos, familia y otros significativos. Este resultado es inesperado, pues la literatura indica que los hombres presentan una percepción de apoyo social familiar y de amigos menor a la que presentan las mujeres (Barra, 2011; Feldman et al., 2008). Nuestros resultados sugieren que tanto los hombres como las mujeres, dadas las características del contexto académico, perciben el apoyo de sus familias y amigos sin grandes diferencias (Dávila et al., 2011).

Además, los resultados indican que solo existen diferencias significativas en el apoyo social percibido de los amigos según el nivel socioeconómico. Específicamente, los sujetos pertenecientes al nivel socioeconómico medio perciben mayor apoyo de los amigos que sus pares pertenecientes al nivel socioeconómico bajo. Al respecto, las investigaciones indican que un bajo nivel socioeconómico aumenta la exclusión y aislamiento social, lo que disminuye el apoyo social percibido de estas personas (OMS, 2003). Así, la pobreza o pertenecer a bajos estratos socioeconómicos está relacionado con una menor percepción de apoyo social (Palomar y Cienfuegos, 2007; Rodríguez, 2010).

En relación con el tercer objetivo, relacionado con determinar el grado de asociación y/o predicción del apoyo social percibido respecto a la presencia de sintomatología de salud mental, se encontró que las tres fuentes de apoyo social percibido (amigos, familia y otros significativos) se asocian de forma significativa e inversa con la sintomatología depresiva, ansiosa y parcialmente con la de estrés. Es decir que, a mayor grado de apoyo social percibido menor era la sintomatología de salud mental asociada. Estos resultados apoyan la tercera hipótesis de investigación referente a que a mayor apoyo social percibido menor sintomatología de salud mental en universitarios chilenos. Estos resultados se asemejan a los antecedentes expuestos en el estudio de Dávila et al. (2011), el cual señala que los universitarios que cuentan con un apoyo social satisfactorio presentan baja sintomatología depresiva; el posible efecto amortiguador del apoyo social podría darse en los momentos en los 
que se produce un aumento de la sintomatología ansiosa en los universitarios. Por otra parte, es llamativo que no se encontrase asociación estadísticamente significativa e inversa entre el apoyo social percibido de los amigos y las puntuaciones en estrés. Probablemente esta ausencia de asociación puede vincularse a que los amigos de la etapa universitaria responden a demandas del ambiente académico que son comunes entre éstos (Feldman et al., 2008) y comparten experiencias y exigencias académicas que propician en ellos también la existencia de esta sintomatología (Barraza et al., 2015; Cova et al., 2007; Halgravez et al., 2016; Marty et al., 2005), lo que probablemente disminuye la posibilidad de dar y recibir apoyo social de otros.

Además, los análisis de regresión múltiple corroboran el efecto de las dimensiones de apoyo social percibido sobre ansiedad, depresión y estrés, destacándose que el apoyo social familiar tal vez podría desempeñar un rol protector de la sintomatología, y no así las otras dos fuentes del apoyo social percibido. A pesar de que las magnitudes de los coeficientes de determinación $\left(R^{2}\right)$ eran bajas (entre .073 y .156), estos valores se asemejan a los obtenidos en otras investigaciones sobre el efecto predictivo del apoyo social percibido (Costa-Requena, Ballester y Gil, 2015). Probablemente la variable apoyo social percibido de la familia, por sí sola no desempeña un papel predictor tan alto como el que podría derivarse en conjunto con otras variables protectoras de la salud mental, lo cual invita a estudiar otras variables, tales como la autoestima o características de personalidad, las cuales influyen en la salud mental y podrían aumentar y complementar el porcentaje de varianza explicado (Costa-Requena, et al., 2015; Novoa y Barra, 2015; San Martin y Barra, 2013).

Más allá del porcentaje de varianza explicado, la evidencia asociada al papel protector del apoyo familiar se relaciona con los hallazgos empíricos que señalan que en la etapa universitaria los jóvenes transitan hacia una autonomía progresiva sin desligarse del apoyo familiar (Barrera-Herrera y Vinet, 2017), y que, cuando éstos se ven sobrepasados por distintas exigencias propias de su etapa, acuden a sus padres en busca de apoyo (Sepúlveda, 2013). Además, desde el punto de vista cultural, el papel fundamental de la familia como factor protector en salud mental puede contextualizarse bajo el análisis de dos conceptos teóricos: el yo interdependiente y el familismo. El primero apunta a que los individuos de las culturas colectivistas, como la chilena, construyen su concepto de sí mismo, el cual requiere de la inclusión de aspectos contextuales o situacionales vinculados con las relaciones interpersonales significativas, como la familia (Harb y Smith, 2008; Markus y Kitayama, 1991). Por su parte, el "familismo" es un concepto que hace referencia a un valor cultural presente en la cultura latina que dicta las normas, expectativas y creencias acerca de la familia, y se compone de lealtad y respeto por el grupo familiar (Stein, González, Cupito, Kiang, y Supple, 2013). Por tanto, aun cuando teóricamente, la adultez emergente define que una de las características centrales de esta etapa es el centrarse en sí mismos por parte de los jóvenes (Arnett, 2000; 2008), para la salud mental se hace relevante y constituyen un factor cultural protector, los vínculos relacionales con la familia.

Por otra parte, al centrar la atención en la prevalencia asociada a las escalas del DASS-21, llama la atención que más del $40 \%$ de los universitarios presentó puntuaciones de riesgo para cada una de las escalas que componen este instrumento. Al comparar este resultado con las investigaciones revisadas previamente, la prevalencia obtenida en este estudio supera el porcentaje obtenido por otras investigaciones con población universitaria (Antúnez y Vinet, 2013; Barraza et al., 2015; Micin y Bagladi, 2011). Estas cifras pueden explicarse por las características propias que presenta la adultez emergente, como, por ejemplo, que es un periodo de inestabilidad (Arnett 2000, 2008), además de un periodo de alta exigencia y estrés (Feldman et al., 2008). Es importante considerar que el instrumento utilizado permitió medir sintomatología y no trastornos de salud mental, por tanto, no todos los universitarios que reportaron este tipo de sintomatologías derivaron en un trastorno clínico. Probablemente, al haber medido trastornos de ansiedad, depresión y estrés, es posible que la prevalencia obtenida hubiese sido más baja o similar a las reportadas en estudios previos.

Respecto a las limitaciones de este estudio, la muestra correspondiente al género masculino era más baja en comparación con las mujeres, por tanto, futuros estudios podrían equiparar la muestra según género para evaluar si los resultados se replican con una muestra balanceada. Otra limitación corresponde al tipo de muestreo utilizado. El muestreo no probabilístico, por conveniencia, permitió incluir a estudiantes de solo dos universidades, por lo que los resultados son representativos de estas dos instituciones y no son generalizables a todos los universitarios chilenos o a jóvenes no universitarios. Futuras investigaciones podrían utilizar un muestreo probabilístico que incluya universidades de otras regiones y de distintos contextos socioeconómicos, para hacer generalizables los resultados, además de complejizar los análisis al posibilitar realizar comparaciones entre distintos tipos de universidades. Por otro lado, también sería relevante poder constatar la presencia de la adultez emergente en jóvenes no universitarios, como jóvenes que trabajan, o 
que no estudian ni trabajan, caracterizando estas variables de salud mental en este nuevo grupo poco estudiado. Otra limitación de este estudio es la ya mencionada utilización del nivel socioeconómico ESOMAR, medida que no necesariamente tiene representatividad con población universitaria. Por este motivo, en estudios similares se recomienda medir el nivel socioeconómico de los estudiantes con deciles, tal como se mencionó previamente.

Futuras líneas de investigación podrían centrarse en estudios longitudinales con el fin de examinar la incidencia de trastornos de salud mental y posibles trayectorias asociadas a la aparición de trastornos o sintomatología de salud mental durante la etapa universitaria. Además, se sugiere continuar con estudios que investiguen otras variables protectoras de la salud mental, tales como la actividad física, la higiene del sueño, la alimentación saludable, las cuales pudieran complementar el efecto protector que tiene el apoyo familiar sobre la salud mental de los adultos emergentes universitarios. Finalmente, como ha quedado expuesto, el contexto universitario es un escenario complejo y, dada la elevada prevalencia de sintomatología de salud mental, se hace urgente elaborar líneas de intervención en torno a la promoción y prevención que incluyan variables protectoras de salud mental como el apoyo social. El universitario es un joven que camina hacia la adultez, en un contexto de estrés, pero también de desarrollo de potencialidades, en donde conjuga su camino amplio y desafiante hacia la autonomía de la mano del apoyo social significativo.

\section{Conflictos de intereses}

Los autores declaran que no existen conflictos de intereses.

\section{Referencias}

ADIMARK (2000). El nivel socioeconómico ESOMAR. Manual de aplicación. Recuperado de http://www.microweb.cl/idm/ documentos/ESOMAR.pdf

Antúnez, Z., y Vinet, E. (2012). Escalas de depresión, ansiedad y estrés (DASS-21): Validación de la versión abreviada en estudiantes universitarios chilenos. Revista Terapia Psicológica, 30(3), 49-55. doi:10.4067/s0718-48082012000300005

Antúnez, Z., y Vinet, E. (2013). Problemas de salud mental en estudiantes de una universidad regional chilena. Revista médica de Chile, 141(2), 209-216. doi:10.4067/S0034-98872013000200010

Aranda, C., y Pando, M. (2013). Conceptualización del apoyo social y las redes de apoyo social. Revista de Investigación en Psicología, 16(1), 233-245. doi:10.15381/rinvp.v16i1.3929

Arnett, J. (2000). Emerging adulthood: A theory of development from the late teens through the twenties. American Psychologist, 55(5), 469-480. doi:10.1037//0003-066X.55.5.469
Arnett, J. (2008). Adolescencia y adultez emergente. Un enfoque cultural. México: Pearson Educación.

Arnett, J., Žukauskienè, R., \& Sugimura, K. (2014). The new life stage of emerging adulthood at ages 18-29 years: implications for mental health. Lancet Psychiatry, 1(7), 569-576. doi:10.1016/S2215-0366(14)00080-7

Arrieta, K., Díaz, S., y González, F. (2013). Síntomas de depresión, ansiedad y estrés en estudiantes de odontología: prevalencia y factores relacionados. Revista Colombiana de Psiquiatría, 42(2), 173-181. doi:10.1016/S0034-7450(13)70004-0

Arrieta, K., Díaz, S., y González, F. (2014). Síntomas de depresión y ansiedad en jóvenes universitarios: prevalencia y factores relacionados. Revista Clínica de Medicina de Familia, 7(1), 14-22. doi:10.4321/s1699-695x2014000100003

Baader, T., Rojas, C., Molina, J., Gotelli, M., Alamo, C., Fierro, C., Venezian, S., y Dittus, P. (2014). Diagnóstico de la prevalencia de trastornos de la salud mental en estudiantes universitarios y los factores de riesgo emocionales asociados. Revista Chilena de Neuro-psiquiatría, 52(3), 167-176. doi: 10.4067/ S0717-92272014000300004

Balanza, S., Morales, I., y Guerrero, J. (2009). Prevalencia de ansiedad y depresión en una población de estudiantes universitarios: Factores académicos y sociofamiliares asociados. Clínica y Salud, 20(2), 177-187.

Barra, E. (2011). Influencia de la autoestima y del apoyo social percibido sobre el bienestar psicológico de estudiantes universitarios chilenos. Diversitas Perspectivas en Psicología, 8(2), 29-38. doi:10.15332/s1794-9998.2012.0001.02

Barraza, R., Muñoz, N., Alfaro, M., Álvarez, A., Araya, V., Villagra, J., y Contreras, A. M. (2015). Ansiedad, depresión, estrés y organización de la personalidad en estudiantes novatos de medicina y enfermería. Revista Chilena de Neuro-psiquiatría, 53(4), 251-260. doi:10.4067/S0717-92272015000400005

Barrera-Herrera, A., y Vinet, E. (2017). Adultez emergente y características culturales de la etapa en universitarios chilenos. Terapia Psicológica, 35(1), 47-56. doi:10.4067/s071848082017000100005

Belhumeur, S., Barrientos Segura, A., y Retana-Salazar, A. (2016). Niveles de estrés de la población estudiantil en Costa Rica. Diferencias en función de las variables nivel socioeconómico, rendimiento académico, nivel académico y zona geográfica. Psychology, Society and Education, 8(1), 13-22. doi: doi.org/10.25115/psye.v8i1.457

Chandavarkar, U., Azzam, A., \& Mathews, C. (2007). Anxiety symptoms and perceived performance in medical students. Depression and Anxiety, 24(2), 103-111. doi:10.1002/da.20185

Costa-Requena, G., Ballester Arnal, R., \& Gil, F. (2015). The influence of demographic and clinical variables on perceived social support in cancer patients. Revista de Psicopatología y Psicología clínica, 20(1), 25-32. doi.org/10.5944/rppc. vol.1.num.1.2015.14404

Cova, F., Alvial, W., Aro, M., Bonifetti, A., Hernández, M., y Rodríguez, C. (2007). Problemas de salud mental en estudiantes de la Universidad de Concepción. Terapia psicológica, 25(2), 105-112. doi: 10.4067/s0718-48082007000200001

Dávila, A., Ruiz, R., Moncada, L., y Gallardo, I. (2011). Niveles de ansiedad, depresión y percepción de apoyo social en estudiantes de odontología de la Universidad de Chile. Revista de Psicología, 20(2), 147-172. doi:10.5354/0719-0581.2012.17937. 
Dutra-Thomé, L. (2013). Emerging Adulthood in southern brazilians from differing socioeconomic status: social and subjective markers. Tesis de Doctorado en Psicología, Universidade Federal do Rio Grande do Sul, Brasil. Recuperado de http:// www.lume.ufrgs.br/bitstream/handle/10183/76534/000886149. pdf? sequence $=1$

Facio, A., \& Micocci, E. (2003). Emerging adulthood in Argentina. New directions in child and adolescent development, 100, 21-31. doi:10.1111/j.1750-8606.2007.00025.x

Feldman, L., Goncalves, L., Puignau, G., Zaragosa., J., Bagés, N., y De Pablo, J. (2008). Relaciones entre estrés académico, apoyo social, salud mental y rendimiento académico en estudiantes venezolanos. Universitas Psychologica, 7(3), 739-751. doi:10.14204/ejrep.35.14053

Ferrel, R., Celis, A., y Hernández, O. (2011). Depresión y factores sociodemográficos asociados en estudiantes universitarios de Ciencias de la Salud de una universidad pública (Colombia). Psicología desde el Caribe, 27, 40-60.

Fierro, D., \& Moreno, A. (2007). Emerging adulthood in mexican and spanish youth: Theories and realities. Journal of Adolescent Research, 22(5), 476-503. doi:10.1177/0743558407305774

Galleguillos, P., Hernández, J., Sepúlveda, C., y Valdés, P. (2016). Reforma a la educación superior: Financiamiento actual y proyecciones. Recuperado de http://www.dipres.gob.cl/598/ articles-154341_doc_pdf.pdf

Halgravez, L., Salinas, J., Martínez, G., y Rodríguez, O. (2016). Percepción de estrés en universitarios, su impacto en el desempeño académico, afrontamiento y apoyo familiar. Revista Mexicana de Estomatología, 3(2), 27-36.

Harb, C., \& Smith, P. (2008). Self-construals across cultures beyond Independence-Interdependence. Journal of Cross-Cultural Psychology, 39(2), 178-197. doi:10.1177/0022022107313861

Lecannelier, F. (2018). El trauma oculto en la infancia. Guía cientificamente informada para padres, educadores y profesionales. Barcelona, España: Penguin Random \& House.

Lovibond, S.H., \& Lovibond, P.F. (1995). Manual for the depression anxiety stress scales. Sydney, Australia: Psychology Foundation of Australia.

Markus, H.R., \& Kitayama, S. (1991). Culture and the self: Implications for cognition, emotion, and motivation. Psychological Review, 98(2), 224-253. doi:10.1037/0033-295x.98.2.224

Marty, M., Lavín, G., Figueroa, M., y Cruz, M. (2005). Prevalencia de estrés en estudiantes del área de la salud de la Universidad de los Andes y su relación con enfermedades infecciosas. Revista chilena de neuro-psiquiatría, 43(1), 25-32. doi:10.4067/S0717-92272005000100004

Matud, M., Guerrero, K., y Matías, R. (2006). Relevancia de las variables sociodemográficas en las diferencias de género en depresión. International Journal of Clinical and Health Psychology, 6(1), 7-21.

Micin, S., y Bagladi, V. (2011). Salud mental en estudiantes universitarios: Incidencia de psicopatología y antecedentes de conducta suicida en población que acude a un servicio de salud estudiantil. Terapia P4sicológica, 29(1), 53-64. doi:10.4067/S0718-48082011000100006

Novoa, C., y Barra, E. (2015). Influencia del apoyo social percibido y los factores de personalidad en la satisfacción vital de estudiantes universitarios. Terapia Psicológica, 33 (3), 239245. doi:10.4067/s0718-48082015000300007
Opazo, T. (04 de mayo de 2019). La silenciosa violencia ABC1. La Tercera. Recuperado de http://interactivo.latercera.com/ violencia-abc1/

Ordorika, I. (2015). Equidad de género en la educación superior. Revista de la Educación Superior, 44(174), 7-18. doi: 10.1016/j.resu.2015.06.001

Organización Mundial de la Salud, OMS (2003). Social determinants of health. The solid facts. Recuperado de http:// www.euro.who.int/__data/assets/pdf_file/0005/98438/ e81384.pdf?ua1

Ortiz-Hernández, L., López-Moreno, S., y Borges, G. (2007). Desigualdad económica y salud mental: revisión de la literatura latinoamericana. Cadernos de Saúde Pública, 23(6), 12551272. doi: 10.1590/S0102-311X2007000600002.

Ortiz, M., y Baeza, M. (2011). Propiedades psicométricas de una escala para medir apoyo social percibido en pacientes chilenos con diabetes tipo 2. Universitas Psychologica, 10(1), 189-196.

Palomar, J., y Cienfuegos, Y. (2007). Pobreza y apoyo social: Un estudio comparativo en tres niveles socioeconómicos. Revista Interamericana de Psicología, 41(2), 177-188.

Pego-Pérez, E., Río-Nieto, M., Fernández, I., y Gutiérrez-García, E. (2018). Prevalencia de sintomatología de ansiedad y depresión en estudiantado universitario del grado en enfermería en la Comunidad Autónoma de Galicia. Revista ENE de Enfermería, 12(2). Recuperado de http://www.ene-enfermeria.org/ ojs/index.php/ENE/article/view/785/ART5v12n2

Pérez, J. C., Cumsille, P., \& Martínez, M. (Marzo, 2008). Construct validity of the Inventory of Dimensions of Emerging Adulthood in a Chilean sample. Sesión de cartel presentado en the biennial meeting of the Society for Research on Adolescence, Chicago, Illinois.

Riveros, F., y Vinaccia-Alpi, S. (2017). Relación entre variables sociodemográficas, patogénicas y salutogénicas, con la calidad de vida en estudiantes universitarios colombianos. Revista de Psicopatología y Psicología Clínica, 22(3), 229-241. https://doi.org/10.5944/rppc.vol.22.num.3.2017.16330

Rodríguez, S. (2010). Relación entre nivel socioeconómico, apoyo social percibido, género y depresión en niños. Interdisciplinaria, 27(2), 261-275.

Román, F., Santibáñez, P., y Vinet, E. V. (2016). Uso de las escalas de depresión ansiedad estrés (DASS-21) como instrumento de tamizaje en jóvenes con problemas clínicos. Acta de Investigación Psicológica, 6(1), 2325-2336. doi: 10.1016/s20074719(16)30053-9

San Martin, J., y Barra, E. (2013). Autoestima, apoyo social y satisfacción vital en adolescentes. Terapia Psicológica, 31(3), 287-291. doi:10.4067/s0718-48082013000300003

Sandín, B., y Chorot, P. (2017). Cuestionario de Sucesos Vitales (CSV). Revista de Psicopatología y Psicología Clínica, 22, 95115.doi:https://doi.org/10.5944/rppc.vol.22.num.2.2017.19729.

Sepúlveda, C. (2013). Perfil de la generación Y chilena. Tesis de pregrado no publicada, Universidad de Chile, Santiago, Chile. Recuperado de http://repositorio.uchile.cl/bitstream/handle/2250/112254/Perfil\%20de $\% 201 \mathrm{a} \% 20$ Generacion $\% 20$ Y\%20Chilena\%20-\%20Carolina\%20Sep\%C3\%BAlveda.pd$\mathrm{f}$ ? sequence $=3$

Sinay, S. (2011). La sociedad de los hijos huérfanos. Buenos Aires, Argentina: Ediciones B. 
Stein, G.L., Gonzalez, L.M., Cupito, A.M., Kiang, L., \& Supple, A.J. (2013) The protective role of Familism in the lives of Latino Adolescents. Journal of Family Issues, 36(10), 12551273. doi:10.1037/a0034811

Stroebe, M., Van Vliet, T., Hewstone, M., \& Willis, H. (2002). Homesickness among students in two cultures: Antecedents and consequences. British Journal of Psychology, 93(2), 147-168. doi:10.1348/000712602162508.

Zimet, G.D., Dahlem, N., Zimet, S.G., \& Farley, G. (1988). The Multidimensional Scale of Perceived Social Support. Journal of Personality Assessment, 52(1), 30-41. doi:10.1207/ s15327752jpa5201_2 
